\title{
The Optimization of Transportation Costs in Logistics Enterprises with Time-Window Constraints
}

\author{
Qingyou Yan and Qian Zhang \\ School of Economics and Management, North China Electric Power University, Beijing 102206, China \\ Correspondence should be addressed to Qian Zhang; zhangqian881111@163.com
}

Received 25 November 2014; Revised 26 March 2015; Accepted 27 March 2015

Academic Editor: Delfim F. M. Torres

Copyright (c) 2015 Q. Yan and Q. Zhang. This is an open access article distributed under the Creative Commons Attribution License, which permits unrestricted use, distribution, and reproduction in any medium, provided the original work is properly cited.

\begin{abstract}
This paper presents a model for solving a multiobjective vehicle routing problem with soft time-window constraints that specify the earliest and latest arrival times of customers. If a customer is serviced before the earliest specified arrival time, extra inventory costs are incurred. If the customer is serviced after the latest arrival time, penalty costs must be paid. Both the total transportation cost and the required fleet size are minimized in this model, which also accounts for the given capacity limitations of each vehicle. The total transportation cost consists of direct transportation costs, extra inventory costs, and penalty costs. This multiobjective optimization is solved by using a modified genetic algorithm approach. The output of the algorithm is a set of optimal solutions that represent the trade-off between total transportation cost and the fleet size required to service customers. The influential impact of these two factors is analyzed through the use of a case study.
\end{abstract}

\section{Introduction}

In a competitive environment, obtaining the maximum profit plays a key role in the success of an enterprise. Logistics costs make up a large portion of the total costs of enterprises but can be reduced through supply chain optimization. Analysis of the logistics costs of enterprises reveals that transportation costs are an important part of the costs of logistics enterprises. Therefore, it is very important to study how transportation costs can be optimized in logistics enterprises.

The transportation costs of logistics enterprises are influenced by the fixed costs and variable costs involved in the transportation process. However, transportation costs are more closely related to time-window constraints, which are governed by customers' arrival times. Logistics enterprises must pay penalties when time-window constraints are violated, and this causes increases in transportation costs.

Many past studies have been dedicated to determining how to achieve the lowest possible transportation cost. For example, McCann [1] addressed two interrelated questions: the optimum size of a vehicle or vessel and the structure of transportation costs with respect to haulage distance. C. Pilot and S. Pilot [2] focused on minimizing the total costs involved in a transportation problem. Jha et al. [3] considered a joint-location inventory problem and minimized the transportation cost involved in a joint inventory location model by using a modified adaptive different evolution algorithm. Chanas and Kuchta [4] proposed what they see as an optimal solution to the transportation problem, which makes use of fuzzy cost coefficients and an algorithm determining the nature of the solution.

As exploration of transportation problems has developed, multiobjective transportation cost problems have emerged. For instance, Prakash et al. [5] drew attention to a cost-time trade-off bulk transportation problem, which they solve by using Pareto optimal solutions. Ojha et al. [6] formulated a multiobjective transportation solution, with fuzzy relations under fuzzy logic. The objectives of their model are the minimization of the total transportation cost and total time for transportation required for the system.

The conditions that force each vehicle to start with each customer at a period specified by that customer are called time-window constraints. Existing literature on transportation problems with time-window constraints has mainly concentrated on vehicle routing problems. Vehicle routing problems, with different variations and generalizations, have been studied for several decades, since the pioneering work of Dantzig and Ramser [7] on a truck dispatching problem. 
Alvarenga et al. [8] proposed a robust heuristic approach to vehicle routing problems with time windows (VRPTW), using travel distance as the main objective through an efficient genetic algorithm and a set partitioning formulation.

Ghoseiri and Ghannadpour [9] presented a new model and solution for multiobjective VRPTW using goal programming and genetic algorithm, in which decision makers specify optimistic aspiration levels to objectives and deviations from those aspirations are minimized. They used a direct interpretation of VRPTW as a multiobjective problem, in which both total required fleet size and total traveling distance were minimized, while capacity and time-window constraints were secured.

Al-Khayyal and Hwang [10] formulated a model for finding the minimum-cost route in a network for a heterogeneous fleet of ships engaged in the pickup and delivery of several liquid bulk products. They showed that the model can be reformulated as an equivalent mixed-integer linear program with a special structure.

Yu et al. [11] proposed a hybrid approach, which consists of ant colony optimization (ACO) and Tabu search, to solve VRPTW.

Chiang and Hsu [12] proposed their own approach to solve a multiobjective vehicle routing problem with time windows. The objectives were to simultaneously minimize the number of vehicles and the total distance. Their approach was based on an evolutionary algorithm and it aims to find a set of Pareto optimal solutions.

Because of the many applications of different vehicle routing problems, a wide variety of researchers have focused on developing solutions to them. Useful techniques for solving general vehicle routing problems can be found in [13-15].

Our analysis of the works described above has shown that existing literature based on accurate algorithms and heuristic algorithms aims to achieve the lowest transportation cost possible. To our knowledge, no study has considered the time-window constraints in the transportation cost model. Time-window constraints increase transportation costs in logistics enterprises, and so it is necessary for logistics enterprises to take the time-window constraints into consideration when making decisions.

This paper presents a biobjective transportation cost model with time-window constraints, which is modeled through modified genetic algorithm. In our study, the simultaneous minimization of fleet size and total transportation cost are considered objective functions.

The model is formulated under the following assumptions:

(1) Time-window constraints are soft, and the time windows specified by customers are elastic.

(2) The service time for a vehicle at its destination is equal to zero.

(3) A route is defined as starting from a depot, going through a number of customers, and ending at the depot. Every customer on the route must be visited only once by one of the vehicles.

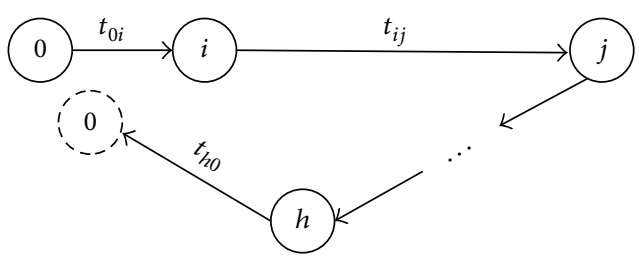

FIGURE 1: A network representation of the transportation process.

\section{Model Formulation}

This paper assumes that a logistics enterprise is the single supplier in a transportation process. The logistics enterprise distributes goods to customers according to the number of orders. The customer number for a route is uncertain, and the vehicle route must be determined in order to optimize transportation cost.

The system of logistics enterprise transportation can be regarded as a simple network. In this network, the start node and end node are both the central depot of the logistics enterprise. Each arc of the network represents the transportation relationship between customers. The number of the arc represents the travel time between the two customers. Let us assume there are $N+1$ customers, $C=\{0,1,2, \ldots, N\}$, and for simplicity denote the depot as customer 0 . Figure 1 presents a network representation of the transportation process.

The transportation cost model in this paper takes timewindow constraints into consideration. Any customer $i$ must be serviced within a predefined time interval $\left[\mathrm{ES}_{i}, \mathrm{LF}_{i}\right]$, limited by an earliest arrival time $\mathrm{ES}_{i}$, and a latest arrival time $\mathrm{LF}_{i}$. Vehicles arriving later than the latest arrival time are penalized, while those arriving earlier than the earliest arrival time have to pay for the inventory cost involved.

In order to formulate the model, the notations in it are defined as follows:

$K$ : the maximum possible size of the fleet,

$C_{k}$ : the transportation cost per unit mass or volume of vehicle $k$,

$C=\{0,1,2, \ldots, N\}:$ the customer set,

$(i, j)$ : the transportation process from customer $i$ to customer $j$,

$t_{i j}$ : the travel time between customer $i$ and customer $j$,

$m_{i}$ : the demand of customer $i$,

$Q_{k}$ : the limited capacity of vehicle $k$,

$x_{i j k}:\left\{\begin{array}{l}1, \text { vehicle } k \text { drives from customer } i \\ \quad \text { to customer } j \\ 0, \text { otherwise, }\end{array}\right.$

$r_{k}$ : the maximum route time allowed for vehicle $k$,

$\left[\mathrm{ES}_{i}, \mathrm{LF}_{i}\right]$ : the predefined time interval that customer $i$ must be serviced within, with $\mathrm{ES}_{i}$ being the earliest arrival time of customer $i$, and $\mathrm{LF}_{i}$ being the latest arrival time of customer $i$, 
$d_{1}$ : the inventory cost that the logistics enterprise has to pay if a vehicle arrives one day earlier than the earliest arrival time,

$d_{2}$ : the penalty cost that the logistics enterprise has to pay if a vehicle arrives one day later than the latest arrival time,

$e_{i k}$ : the arrival time in advance for vehicle $k$ at node $i$,

$f_{i k}$ : the delayed arrival time for vehicle $k$ at node $i$,

$T_{i k}$ : the real arrival time for vehicle $k$ at node $i$.

There are two objectives in the transportation cost model. One is to minimize the transportation cost, and the other is to minimize the fleet size used to serve the customers. The model has four constraints. This paper observes vehicle capacity constraints and time-window constraints. In it, each customer is served exactly once and each vehicle starts its journey from a depot and ends at the depot.

The transportation cost model in this paper can be written as

$$
\begin{gathered}
\operatorname{Min} \sum_{i=1}^{N} m_{i} \sum_{j=0, i \neq j}^{N} \sum_{k=1}^{K} x_{i j k} \times C_{k}+\sum_{i=1}^{N} \sum_{k=1}^{K} e_{i k} \times d_{1} \\
+\sum_{i=1}^{N} \sum_{k=1}^{K} f_{i k} \times d_{2} \\
\operatorname{Min} \sum_{k=1}^{K} \sum_{j=1}^{N} x_{0 j k},
\end{gathered}
$$

subject to

$$
\begin{array}{r}
\sum_{j=1, j \neq i}^{N} x_{i j k}=\sum_{j=1, j \neq i}^{N} x_{j i k} \leq 1 \\
\text { for } i=\{0,1, \ldots, N\}, k=\{1,2, \ldots, K\}, \\
\sum_{k=1}^{K} \sum_{j=0, j \neq i}^{N} x_{i j k}=1 \quad \text { for } i=\{1,2, \ldots, N\}, \\
\sum_{k=1}^{K} \sum_{i=0, i \neq j}^{N} x_{i j k}=1 \quad \text { for } j=\{1,2, \ldots, N\}, \\
\sum_{i=1}^{N} m_{i} \sum_{j=0, j \neq i}^{N} x_{i j k} \leq Q_{k} \quad \text { for } k=\{1,2, \ldots, K\}, \\
\sum_{i=0}^{N} \sum_{j=0, j \neq i}^{N} x_{i j k} t_{i j} \leq r_{k} \quad \text { for } k=\{1,2, \ldots, k\}, \\
\mathrm{ES}_{0}=\mathrm{LF}_{0}=0, \\
\sum_{k=1}^{K} \sum_{i=0, i \neq j}^{N} x_{i j k}\left(T_{i k}+t_{i j}\right)=T_{j k} \quad \text { for } j=\{i, \ldots, N\},
\end{array}
$$
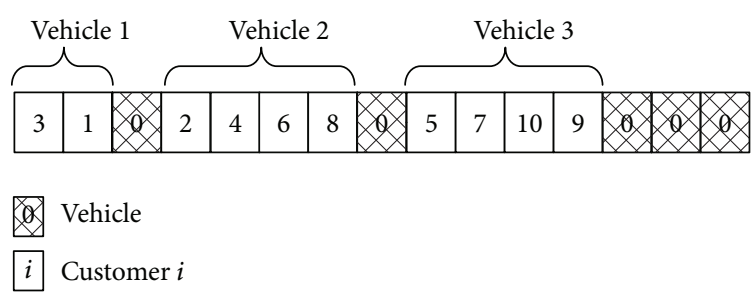

FIGURE 2: Representation of the individual integer code.

$$
\begin{gathered}
\mathrm{ES}_{i} \leq \sum_{k=1}^{K} T_{i k}+e_{i}-f_{i} \leq \mathrm{LF}_{i}, \\
e_{i} \times f_{i}=0 .
\end{gathered}
$$

Constraint (2) specifies that every route starts and ends at the central depot. Constraints (3) and (4) define that every customer node is visited only once by one vehicle. Constraint (5) is the capacity constraint. Constraint (6) is the maximum travel time constraint. Constraints (7)-(10) define the time windows.

\section{Modified Genetic Algorithm}

As mentioned before, Ghoseiri and Ghannadpour [9] studied a vehicle routing problem with time windows and presented a genetic algorithm (GA) to solve it. In this section, we modify the presented GA to be used in the multiobjective optimization model above, by further considering the total transportation cost and time-window constraints. The specific content is described below.

A GA starts with a set of chromosomes referred to as the initial population. Each chromosome represents a solution to the problem, and the initial population is randomly generated. A selection mechanism is then used to select prospective parents based on their fitness, computed by the evaluation function. The selected parent chromosomes are then recombined via the crossover operator to create a potential new population. The next step is to mutate a small number of newly obtained chromosomes, in order to introduce a level of randomness that prevents the GA from converging to a local optimum.

3.1. Chromosome Representation and the Initial Population. To solve a transportation cost model, each individual is usually represented by just one chromosome, which is a chain of integers (as discussed by Ghoseiri and Ghannadpour [9]). In this representation, each vehicle identifier is represented by 0 and is also a separator between two different routes. There are $K-1$ vehicle identifiers in the chromosome, which can divide the chromosome into $K$ routes. The customer identifiers are represented by $i(i=1,2, \ldots, N)$, and a string of customer identifiers represents the sequence of deliveries that a vehicle must cover during its route. Figure 2 shows a representation of a possible solution with 10 customers and 6 vehicles. The route of vehicle 1 is $3 \rightarrow 1$, the second route is $2 \rightarrow 4 \rightarrow 6 \rightarrow 8$, and the third is $5 \rightarrow 7 \rightarrow 10 \rightarrow 9$. There are no travel tasks for vehicles 4,5 , and 6 . 
An initial population is built such that each individual must at least be a feasible candidate solution (i.e., every route in the initial population must be feasible). In this paper, an individual is feasible when the limited capacity of the vehicle and the maximum route time allowed are satisfied. Based on this, the population initialization algorithm can be written as follows.

Step 1. Determine the set of all unscheduled customers $\alpha=$ $\{1,2, \ldots, N\}$ and the maximal size of fleets $K$. Denote $Z_{k}$ and $R_{k}$ as the rest capacity and travel time of vehicle $k(k=$ $1,2, \ldots, K)$, respectively. Let $\beta_{k}$ be the set consisting of all customers served by vehicle $k$. Initialize $Z_{k}=Q_{k}, R_{k}=r_{k}$, and $\beta_{k}=\varnothing$ for all $k=1,2, \ldots, K$.

Step 2. Randomly select an unscheduled customer $i$ who has higher demand than others (i.e., $i \in\left\{r \mid m_{r}=\max _{r \in \alpha}\left\{m_{r}\right\}\right\}$ ). Let $c_{k}(k=1,2, \ldots, K)$ be the last customer in the set $\beta_{k}$ (if $\beta_{k}=\varnothing$, then $c_{k}$ represents the central depot). Then, determine the set $\chi$, which consists of all vehicles whose rest capacities and rest route times are sufficient to serve customer $i$ (i.e., $\chi=\left\{k \mid m_{i} \leq Z_{k}, t_{i_{k} i} \leq R_{k}, k=1,2, \ldots, K\right\}$ ). Finally, randomly choose a vehicle $\bar{k}$ from the set $\chi$ to serve customer $i$ and update $\beta_{\bar{k}}=\left\{\beta_{\bar{k}}, i\right\}, \alpha=\alpha \backslash\{i\}, Z_{\bar{k}}=Z_{\bar{k}}-m_{i}$, and $R_{\bar{k}}=R_{\bar{k}}-t_{c_{\bar{k}}}$.

Step 3. If $\alpha=\varnothing$, go to the next step; otherwise, go to Step 2 .

Step 4. Rearrange the sequence of customers in set $\beta_{k}$ and randomly generate a feasible travel route for vehicle $k$, denoted by $\mathrm{FR}_{k}$, for all $k=1,2, \ldots, K$. Finally, a complete individual can be represented as $\left\{\mathrm{FR}_{1}, 0, \mathrm{FR}_{2}, 0, \ldots, \mathrm{FR}_{K}\right\}$.

Using the above presentation method, the individual shown in Figure 2 is coded as $\{\{3,1\}, 0,\{2,4,6,8\}, 0,\{5,7$, $10,9\}, 0,0,0\}$.

3.2. Selection. A feasible solution (i.e., individual solution) for the models is said to dominate another solution if it is not worse with regard to any of the objectives and clearly better with regard to at least one. In the current problem, a feasible solution $(C, N)$ is said to dominate another solution $\left(C^{\prime}, N^{\prime}\right)$ if both $C$ and $N$ are smaller than or equal to $C^{\prime}$ and $N^{\prime}$, where $C$ and $C^{\prime}$ denote the total transportation costs and $N$ and $N^{\prime}$ denote the total number of vehicles. The feasible solution $(C, N)$ is said to be a nondominated solution (or a Pareto solution), if there is no other solution that can dominate it. Based on this nondomination criterion, this paper evaluates and selects the individuals through the following procedure.

Step 1. Calculate the objective function values for all individuals, where the total transportation costs are determined by (11), and total numbers of vehicles are determined directly by decoding the chromosome.

Step 2. Assign a rank value to each individual, depending on its position within the population. The smaller the number of solutions that dominate an individual, the smaller the rank of the individual. If we suppose that, for a given generation $t$, an individual $k$ is dominated by the number of $d_{k}$ of individuals in the considered population, its rank will be determined as follows:

$$
\operatorname{rank}(k, t)=1+d_{t}
$$

It is easy to determine that all nondominated solutions will have a rank equal to 1 . Note also that, for a given individual, this metric may vary across generations because of the population distribution changes.

Step 3. Compute the fitness value of an individual $k$ in a population at a generation $t$ by calculating the following (as discussed by Elloumi and Fortemps [16]):

$$
f(k, t)=\frac{1}{\operatorname{rank}(k, t)} .
$$

Step 4. Randomly select $\mathrm{POP} / 2$ pairs of individuals, by roulette selection, to undergo forthcoming evolutionary operations, where POP denotes the number of individuals within a population. The selection probability of individual $k$ in generation $t$ is given by the following:

$$
p(k, t)=\frac{f(k, t)}{\sum_{l=1}^{\mathrm{POP}} f(l, t)} .
$$

3.3. Crossover. This paper employs a problem-specific best cost-route crossover (as discussed by Ombuki et al. [13]), with the probability of crossover $p_{\text {cross }}$. This aims to simultaneously minimize the fleet size and the total transportation cost, while also assessing the feasibility of constraints. For any pair of individuals randomly selected through the selection operation above, one is called the "father" and is denoted by $I^{F}=\left(\beta_{1}^{F}, 0, \beta_{2}^{F}, 0, \ldots, \beta_{K}^{F}\right)$. Another is termed the "mother" and is denoted by $I^{M}=\left(\beta_{1}^{M}, 0, \beta_{2}^{M}, 0, \ldots, \beta_{K}^{M}\right)$.

First, a random route from the father is selected. Second, all of the genes in the selected route are removed from the mother, and the remaining chromosome is directly passed on to the son, $I^{S}$. Third, each of the removed genes is reinserted into the son's line, based on the minimum cost principle, and the whole offspring $I^{S}$ is then obtained by the end of this step. We proceed similarly for the daughter, $I^{D}$, but replace the father with the mother and vice versa.

The pseudocode for generating the son $I^{S}$ can be described as shown in Procedure 1.

In Procedure 1, length $\left(I^{D}\right)$ is the number of genes of $I^{D}$. $I_{i \rightarrow j}^{D}$ is the chromosome of $I^{D}$ between position $i$ and position $j$.

3.4. Mutation. The mutation operator is applied to newly generated individuals with a probability of mutation $p_{\text {mut }}$. First, we randomly choose two positions, $q_{\text {mut1 }}$ and $q_{\text {mut2 }}$, such that $1 \leq q_{\text {mut } 1}<q_{\text {mut } 2} \leq N+K-1, q_{\text {mut } 1} \neq 0$, and $q_{\text {mut2 }} \neq 0$ (recall that $N$ and $K$ are the numbers of customers and vehicles, resp.). Then, we check whether customers in positions $q_{\text {mut } 1}$ and $q_{\text {mut } 2}$ can be permuted. In other words, if the change does not result in infeasibility, we can permute the customers. Otherwise, we choose another position, $q_{\text {mut2 }}^{\prime}$. 


\section{BEGIN:}

(1) Randomly generate a number $q_{\text {cross }}$, such that $1 \leq q_{\text {cross }} \leq K$ and $\beta_{\text {cross }}^{F} \neq \varnothing$;

(2) Let $I^{D}=I^{M} \backslash\left\{\beta_{\text {cross }}^{F}\right\}$;

(3) Randomly select a gene $g^{F}$ in $\beta_{\text {cross }}^{F}$;

(4) FOR $i=1$ TO length $\left(I^{D}\right)$;

(5) Generate the partial schedule $P_{i}=\left(I_{1 \rightarrow i-1}^{D}, g^{F}, I_{i \rightarrow \text { end }}^{D}\right)$;

(6) Compute the total transportation cost $C_{i}$ of $P_{i}$. If $P_{i}$ is infeasible, let $C_{i}=$ a sufficiently large number;

(7) Let $i^{*}=\min _{i}\left\{r \mid C_{r}\right\}$ and reinsert gene $g^{F}$ in the position $i^{*}$ of $I^{D}$;

(8) Update $I^{D}=\left(I_{1 \rightarrow i^{*}-1}^{D}, g^{F}, I_{i^{*} \rightarrow \text { end }}^{D}\right)$ and $\beta_{\text {cross }}^{F}=\beta_{\text {cross }}^{F} \backslash g^{F} ;$ ENDFOR

(9) If $\beta_{\text {cross }}^{F} \neq \varnothing$, return to Step (3); otherwise, terminate the procedure.

Procedure 1

BEGIN

Input: all necessary data related to customers and vehicles

GA parameters: POP, $p_{\text {cross }}, p_{\text {mut }}$

Generate a new population of individuals

While maximal iteration number is not met DO

Evaluate the individuals

Select the better individuals

Generate the offspring population using evolutionary operators

END

Output: a set of non-dominated solutions

Procedure 2

TABLE 1: The situation of vehicles in the logistics enterprise S.

\begin{tabular}{lccc}
\hline Vehicle model & $Q_{k}$ & $C_{k}$ & Quantity \\
\hline $\mathrm{a}$ & 15 ton $/ 59 \mathrm{~m}^{3}$ & 0.2 yuan $/ \mathrm{kg}$ & 2 \\
$\mathrm{~b}$ & 2 ton $/ 15 \mathrm{~m}^{3}$ & 0.3 yuan $/ \mathrm{kg}$ & 1 \\
$\mathrm{c}$ & $25 \mathrm{ton} / 77 \mathrm{~m}^{3}$ & 0.15 yuan $/ \mathrm{kg}$ & 2 \\
$\mathrm{~d}$ & 40 ton $/ 100 \mathrm{~m}^{3}$ & 0.1 yuan $/ \mathrm{kg}$ & 2 \\
\hline
\end{tabular}

We repeat the procedure until two customers are permuted or until a certain number of unsuccessful attempts are made.

Finally, the main process of proposed GA can be described as shown in Procedure 2.

\section{Experimental Results and Comparisons}

A logistics enterprise named $\mathrm{S}$ has 10 vehicles, the situation of which is shown in Table 1 . S needs to deliver goods to 10 customers. Table 2 shows the travel time for S's logistics enterprise. The positions and demands of the customers can be seen in Figure 3 and Table 3. Table 4 presents the timewindows constraints for each customer. When a vehicle's arrival time is one day earlier than the earliest permitted arrival time, the inventory cost is equal to 0.44 yuan/(ton $\times$ day). The penalty cost, when the vehicle's arrival time is one day later than the latest permitted arrival time, is 4,000 yuan/weekday. The maximum travel time for each route is 30 weekdays.

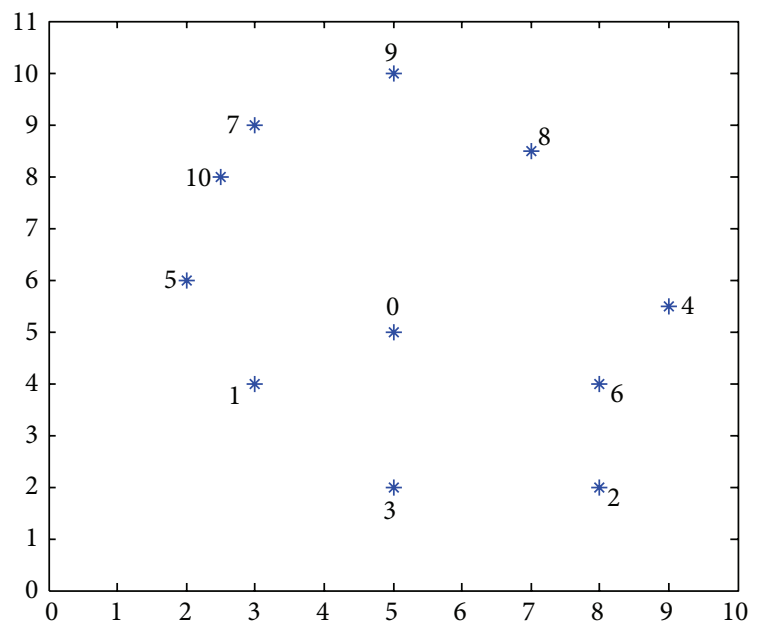

FIgURE 3: The position relationship of the customers.

In real life, it is important to confirm the trade-off between fleet sizes and total transportation costs of logistics enterprises. These two aspects may positively correlate with each other. In other words, a higher transportation cost may be incurred if more vehicles are involved. At the same time, these two values may conflict with each other. Such situations are difficult to determine when using the classical approach but are easily analyzed with our suggested approach. 
TABLE 2: The travel time of S logistics enterprise (day).

\begin{tabular}{llllllllllll}
\hline Travel time & 0 & 1 & 2 & 3 & 4 & 5 & 6 & 7 & 8 & 9 \\
\hline Depot 0 & 0 & 2 & 3 & 2 & 3 & 3 & 3 & 4 & 4 & 5 \\
Customer 1 & - & 0 & 3 & 3 & 2 & 2 & 3 & 3 & 5 & 5 & 3 \\
Customer 2 & - & - & 0 & 4 & 3 & 2 & 1 & 2 & 4 & 3 & 2 \\
Customer 3 & - & - & - & 0 & 4 & 4 & 5 & 5 & 6 & 6 & 4 \\
Customer 4 & - & - & - & - & 0 & 2 & 2 & 3 & 5 & 5 & 2 \\
Customer 5 & - & - & - & - & - & 0 & 2 & 2 & 5 & 5 & 1 \\
Customer 6 & - & - & - & - & - & - & 0 & 1 & 4 & 4 & 1 \\
Customer 7 & - & - & - & - & - & - & - & 0 & 5 & 3 & 1 \\
Customer 8 & - & - & - & - & - & - & - & - & 0 & 5 & 5 \\
Customer 9 & - & - & - & - & - & - & - & - & - & 0 & 4 \\
Customer 10 & - & - & - & - & - & - & - & - & - & - & 0 \\
\hline
\end{tabular}

TABle 3: The demand of customers.

\begin{tabular}{lc}
\hline Customer & Demand \\
\hline Customer 1 & $3000 \mathrm{~kg}$ \\
Customer 2 & $10000 \mathrm{~kg}$ \\
Customer 3 & $5000 \mathrm{~kg}$ \\
Customer 4 & $2000 \mathrm{~kg}$ \\
Customer 5 & $2000 \mathrm{~kg}$ \\
Customer 6 & $10000 \mathrm{~kg}$ \\
Customer 7 & $8000 \mathrm{~kg}$ \\
Customer 8 & $5000 \mathrm{~kg}$ \\
Customer 9 & $7000 \mathrm{~kg}$ \\
Customer 10 & $20000 \mathrm{~kg}$ \\
\hline
\end{tabular}

TABLE 4: The time windows constraints of each customer.

\begin{tabular}{lc}
\hline Customer & Time windows \\
\hline Customer 1 & {$[1,2]$} \\
Customer 2 & {$[2,4]$} \\
Customer 3 & {$[3,5]$} \\
Customer 4 & {$[4,8]$} \\
Customer 5 & {$[3,10]$} \\
Customer 6 & {$[11,15]$} \\
Customer 7 & {$[3,6]$} \\
Customer 8 & {$[7,12]$} \\
Customer 9 & {$[4,10]$} \\
Customer 10 & {$[2,7]$} \\
\hline
\end{tabular}

In this paper, the proposed GA is run under the MATLAB development environment and performed on a computer with a $2 \mathrm{GHz}$ Intel dual-core processor with $2 \mathrm{~GB}$ RAM, running Windows 7 . We tuned the algorithm parameters, including population size, max iteration, mutation rate, and crossover rate, by using crossover values of $\{0.6,0.7,0.8,0.9\}$ and mutation rates of $\{0.1,0.2,0.3,0.4\}$. After many trials, the population size, crossover rate, mutation rate, and max iteration were set to $50,0.8,0.4$, and 500 , respectively. The example case was solved and repeated 30 times, and the average CPU time was 102 seconds. The best results for the GA are listed in Tables 5 and 6.
The results with time-window constraints are shown in Table 5, and the results without time-window constraints are shown in Table 6. Table 5 shows that the transportation cost of a solution is reduced as the number of vehicles is increased. For example, when the fleet size is 2 , the total transportation cost is 65,320 yuan, while when the fleet size is 3 , the total transportation cost is 34,581 yuan. When the fleet size is increased by 1 , the total transportation cost is thus reduced by $47.06 \%$ (from 65,320 to 34,581 ).

Comparing the results of Tables 5 and 6 , we can find that the total transportation cost with time-window constraints is higher than the transportation cost without them. In particular, when the fleet size is equal to 2 , the gap between the two costs can be as high as 26,520 yuan. Tables 5 and 6 present the different vehicle routes for the two results. This situation can be explained by the real transportation process. In reality, in daily life, some customers determine the goods arrival time interval according to their demand, and this arrival time interval is called a time window. If a customer is serviced before the earliest arrival time, extra inventory costs are incurred. If the customer is serviced after the latest arrival time, penalty costs have to be paid. Therefore, timewindow constraints may influence transportation costs and vehicle routes. It must be mentioned that decision-makers may make the wrong decisions when they ignore the effects of time-window constraints, which results in resource wasting and customer dissatisfaction.

The vehicle routes with time-window constraints of different fleet sizes are shown in Figure 4. When the fleet size is equal to 2 , there are two routes, when the fleet size is equal to 3 , there are three routes, and so on. When the fleet size is increased by 1 , the vehicle routes also increase by 1 . Note that the results in Table 5 and Figure 4 are clearly comparable, and the decision-maker of $\mathrm{S}$ can decide which vehicle route is more preferable, based on specific preferences. For instance, if the decision-maker wants fewer transfers to occur, in order to use vehicle space efficiently, the two-vehicle route may be more reasonable. If the decision maker wants to lower cost, the six-vehicle route may be more reasonable.

The results of this example case allow us to obtain information on the trade-off relationship between fleet size and total transportation cost, as shown in Figure 5. It can be 
TABLE 5: The results with time-window constraints.

\begin{tabular}{|c|c|c|c|}
\hline Fleet size & Limited capacity & The vehicle route & Total transportation cost \\
\hline 2 & {$[40,40]$} & $\begin{array}{l}0 \rightarrow 1 \rightarrow 5 \rightarrow 10 \rightarrow 7 \rightarrow 9 \rightarrow 0 \\
0 \rightarrow 3 \rightarrow 2 \rightarrow 6 \rightarrow 4 \rightarrow 8 \rightarrow 0\end{array}$ & 65320 \\
\hline 3 & {$[40,40,25]$} & $\begin{array}{l}0 \rightarrow 10 \rightarrow 7 \rightarrow 9 \rightarrow 8 \rightarrow 0 \\
0 \rightarrow 2 \rightarrow 6 \rightarrow 4 \rightarrow 5 \rightarrow 0 \\
0 \rightarrow 1 \rightarrow 3 \rightarrow 0\end{array}$ & 34581 \\
\hline 4 & {$[40,40,25,25]$} & $\begin{array}{l}0 \rightarrow 2 \rightarrow 6 \rightarrow 8 \rightarrow 0 \\
0 \rightarrow 10 \rightarrow 7 \rightarrow 9 \rightarrow 0 \\
0 \rightarrow 1 \rightarrow 5 \rightarrow 4 \rightarrow 0 \\
0 \rightarrow 3 \rightarrow 0\end{array}$ & 30533 \\
\hline 5 & {$[40,40,25,25,15]$} & $\begin{array}{l}0 \rightarrow 10 \rightarrow 7 \rightarrow 9 \rightarrow 0 \\
0 \rightarrow 2 \rightarrow 6 \rightarrow 5 \rightarrow 0 \\
0 \rightarrow 1 \rightarrow 4 \rightarrow 0 \\
0 \rightarrow 8 \rightarrow 0 \\
0 \rightarrow 3 \rightarrow 0\end{array}$ & 29440 \\
\hline 6 & {$[40,40,15,15,25,25]$} & $\begin{array}{l}0 \rightarrow 10 \rightarrow 7 \rightarrow 9 \rightarrow 0 \\
0 \rightarrow 2 \rightarrow 6 \rightarrow 4 \rightarrow 0 \\
0 \rightarrow 8 \rightarrow 0 \\
0 \rightarrow 3 \rightarrow 0 \\
0 \rightarrow 1 \rightarrow 0 \\
0 \rightarrow 5 \rightarrow 0\end{array}$ & 29240 \\
\hline 7 & {$[40,40,25,25,15,15,2]$} & $\begin{array}{l}0 \rightarrow 10 \rightarrow 7 \rightarrow 9 \rightarrow 0 \\
0 \rightarrow 2 \rightarrow 6 \rightarrow 4 \rightarrow 0 \\
0 \rightarrow 8 \rightarrow 0 \\
0 \rightarrow 3 \rightarrow 0 \\
0 \rightarrow 1 \rightarrow 0 \\
0 \rightarrow 5 \rightarrow 0\end{array}$ & 29240 \\
\hline
\end{tabular}

TABLE 6: The results without time-window constraints.

\begin{tabular}{|c|c|c|c|}
\hline Fleet size & Limited capacity & The vehicle route & Total transportation cost \\
\hline 2 & {$[40,40]$} & $\begin{array}{l}0 \rightarrow 2 \rightarrow 6 \rightarrow 7 \rightarrow 9 \rightarrow 8 \rightarrow 0 \\
0 \rightarrow 10 \rightarrow 5 \rightarrow 1 \rightarrow 3 \rightarrow 4 \rightarrow 0\end{array}$ & 38800 \\
\hline 3 & {$[40,40,25]$} & $\begin{array}{l}0 \rightarrow 10 \rightarrow 7 \rightarrow 9 \rightarrow 4 \rightarrow 0 \\
0 \rightarrow 2 \rightarrow 6 \rightarrow 8 \rightarrow 0 \\
0 \rightarrow 3 \rightarrow 1 \rightarrow 5 \rightarrow 0\end{array}$ & 33350 \\
\hline 4 & {$[40,40,25,25]$} & $\begin{array}{l}0 \rightarrow 6 \rightarrow 2 \rightarrow 8 \rightarrow 0 \\
0 \rightarrow 10 \rightarrow 7 \rightarrow 9 \rightarrow 0 \\
0 \rightarrow 1 \rightarrow 5 \rightarrow 4 \rightarrow 0 \\
0 \rightarrow 3 \rightarrow 0\end{array}$ & 31000 \\
\hline 5 & {$[40,40,25,25,15]$} & $\begin{array}{l}0 \rightarrow 10 \rightarrow 7 \rightarrow 9 \rightarrow 0 \\
0 \rightarrow 6 \rightarrow 2 \rightarrow 5 \rightarrow 0 \\
0 \rightarrow 8 \rightarrow 0 \\
0 \rightarrow 1 \rightarrow 4 \rightarrow 0 \\
0 \rightarrow 3 \rightarrow 0\end{array}$ & 29400 \\
\hline 6 & {$[40,40,15,15,25,25]$} & $\begin{array}{l}0 \rightarrow 2 \rightarrow 6 \rightarrow 4 \rightarrow 0 \\
0 \rightarrow 10 \rightarrow 7 \rightarrow 9 \rightarrow 0 \\
0 \rightarrow 3 \rightarrow 0 \\
0 \rightarrow 8 \rightarrow 0 \\
0 \rightarrow 5 \rightarrow 0 \\
0 \rightarrow 1 \rightarrow 0\end{array}$ & 29200 \\
\hline 7 & {$[40,40,25,25,15,15,2]$} & $\begin{array}{l}0 \rightarrow 10 \rightarrow 7 \rightarrow 5 \rightarrow 0 \\
0 \rightarrow 6 \rightarrow 2 \rightarrow 9 \rightarrow 0 \\
0 \rightarrow 8 \rightarrow 0 \\
0 \rightarrow 3 \rightarrow 0 \\
0 \rightarrow 1 \rightarrow 0 \\
0 \rightarrow 4 \rightarrow 0\end{array}$ & 29200 \\
\hline
\end{tabular}



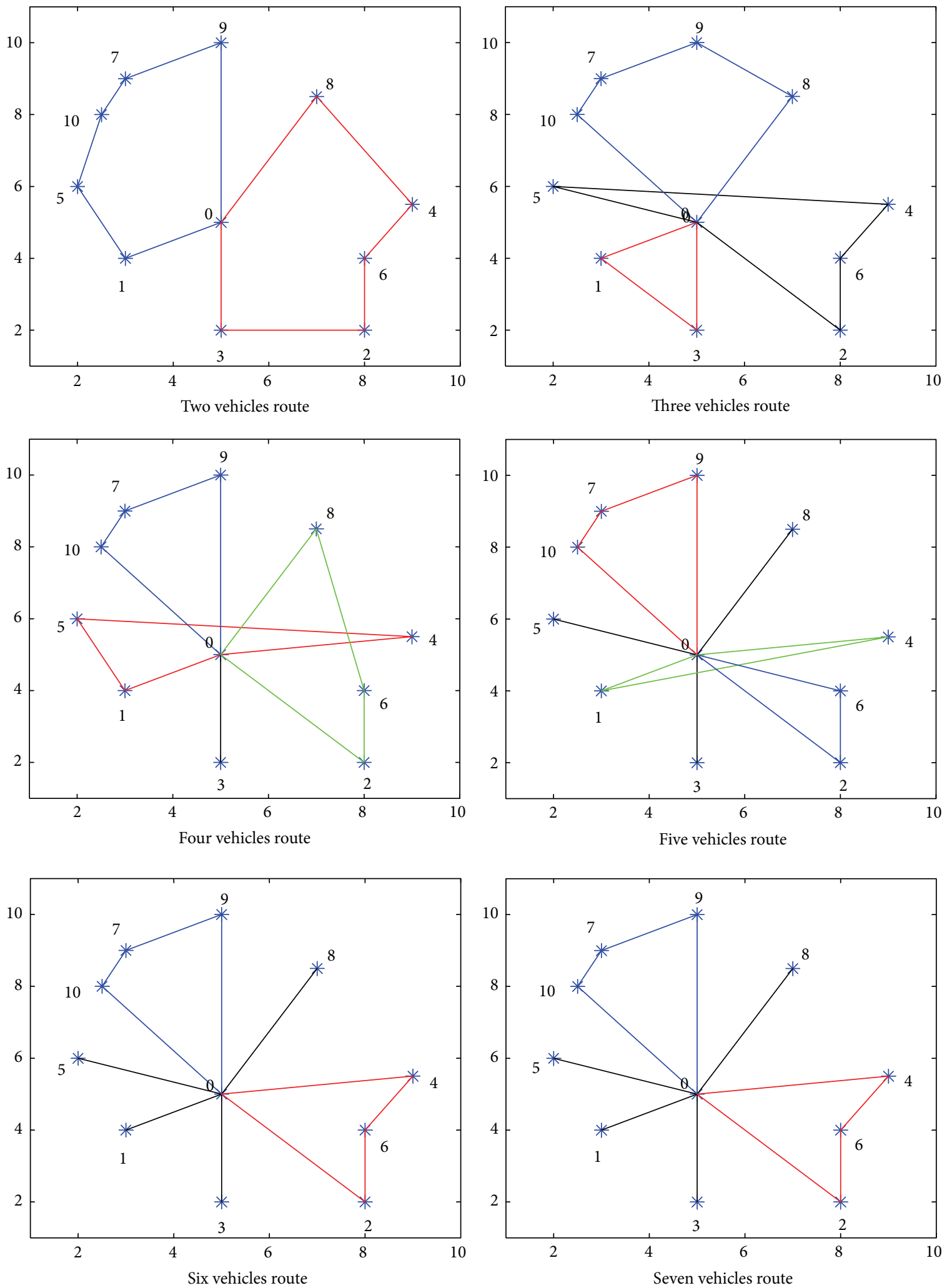

FIGURE 4: The vehicle routes with time-window constraints of different fleet sizes. 


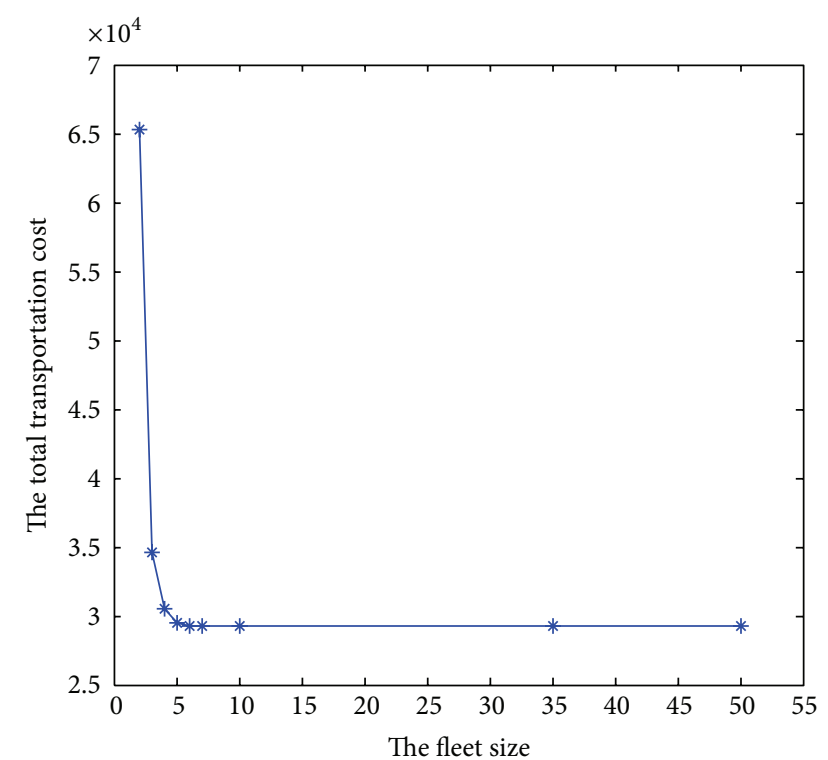

FIGURE 5: The trade-off relationship between fleet size and total transportation cost.

found that these two objectives conflict with each other, and the total transportation cost is reduced as the fleet size is increased. The reason for this is that the transportation weight is a dominant part of transportation cost. However, transportation cost reduction decreases gradually with the increase of fleet size. When the fleet size is six, the transportation cost is at a minimum and will not change further if the fleet size keeps increasing. This situation may be due to fuel consumption, driver salaries, or vehicle maintenance costs, which increase transportation cost.

Based on these findings, this paper emphasizes that the number of vehicles involves separate objectives, beyond the total cost of traveling. This is because there is a cost associated with having more vehicles, and considering this in different cases is important. In some cases when a vehicle and its associated costs (namely, manpower costs, fuel consumptions cost, etc.) are negligible, the routing plan will be irrational. This model represents a range of possible answers, with different numbers of vehicles and costs, with which a decisionmaker can decide which kind of solution is preferable.

\section{Conclusion}

In this paper, we have considered a transportation cost problem with time-window constraints as a biobjective problem in which the size of a fleet of vehicles and total transportation costs are minimized, while capacity and time-window constraints are not violated. This paper uses a modified algorithm approach to solve this transportation cost problem. The proposed GA employs a string of customer identifiers, which represent the sequence of deliveries that must cover a vehicle during its route. Each vehicle identifier represents a separator between two different routes in the chromosome. A selection mechanism is then used to select prospective parents based on their fitness, as computed by an evaluation function. In this paper, the selected parent chromosomes are then recombined via a crossover operator to create a potential new population. A special mutation is applied to introduce a level of randomness that will preserve the GA from converging to a local optimum.

Finally, the algorithm is applied to solve an enterprise transportation problem for S logistics. In the last part of this paper, we compare two results (results with time-window constraints and results without time-window constraints). Based on the gap between these two situations, we find that the total transportation cost with time-window constraints is higher than the transportation cost without timewindow constraints. In addition, time-window constraints also change vehicle routes during transportation.

Through an analysis of the case, we also find that total transportation cost and fleet size conflict with one another and that the total transportation cost is reduced as fleet size is increased. The results with time-window constraints are clearly comparable, and the decision-maker for S can decide which vehicle route is more preferable based on specific preferences. Thus, it is important for logistics enterprises' decision-makers to ensure that the sizes of their fleets of vehicles are appropriate.

\section{Conflict of Interests}

The authors declare that there is no conflict of interests regarding the publication of this paper.

\section{Acknowledgments}

The authors are indebted to Delfim F. M. Torres (Editor) and the two anonymous referees of the journal for their most helpful comments ever. This research is supported by the project of the production-study-building for joint training of graduate students in Beijing.

\section{References}

[1] P. McCann, "A proof of the relationship between optimal vehicle size, haulage length and the structure of distance-transport costs," Transportation Research Part A: Policy and Practice, vol. 35, no. 8, pp. 671-693, 2001.

[2] C. Pilot and S. Pilot, "A model for allocated versus actual costs in assignment and transportation problems," European Journal of Operational Research, vol. 112, no. 3, pp. 570-581, 1999.

[3] A. Jha, K. Somani, M. K. Tiwari, F. T. S. Chan, and K. J. Fernandes, "Minimizing transportation cost of a joint inventory location model using modified adaptive differential evolution algorithm," International Journal of Advanced Manufacturing Technology, vol. 60, no. 1-4, pp. 329-341, 2012.

[4] S. Chanas and D. Kuchta, "A concept of the optimal solution of the transportation problem with fuzzy cost coefficients," Fuzzy Sets and Systems, vol. 82, no. 3, pp. 299-305, 1996.

[5] S. Prakash, P. Kumar, B. V. N. S. Prasad, and A. Gupta, "Pareto optimal solutions of a cost-time trade-off bulk transportation problem," European Journal of Operational Research, vol. 188, no. 1, pp. 85-100, 2008. 
[6] A. Ojha, S. K. Mondal, and M. Maiti, “Transportation policies for single and multi-objective transportation problem using fuzzy logic," Mathematical and Computer Modelling, vol. 53, no. 9-10, pp. 1637-1646, 2011.

[7] G. B. Dantzig and R. H. Ramser, "The truck dispatching problem," Management Science, vol. 6, no. 1, pp. 80-91, 1959.

[8] G. B. Alvarenga, G. R. Mateus, and G. de Tomi, "A genetic and set partitioning two-phase approach for the vehicle routing problem with time windows," Computers \& Operations Research, vol. 34, no. 6, pp. 1561-1584, 2007.

[9] K. Ghoseiri and S. F. Ghannadpour, "Multi-objective vehicle routing problem with time windows using goal programming and genetic algorithm," Applied Soft Computing Journal, vol. 10, no. 4, pp. 1096-1107, 2010.

[10] F. Al-Khayyal and S.-J. Hwang, "Inventory constrained maritime routing and scheduling for multi-commodity liquid bulk. Part I. Applications and model," European Journal of Operational Research, vol. 176, no. 1, pp. 106-130, 2007.

[11] B. Yu, Z. Z. Yang, and B. Z. Yao, "A hybrid algorithm for vehicle routing problem with time windows," Expert Systems with Applications, vol. 38, no. 1, pp. 435-441, 2011.

[12] T.-C. Chiang and W.-H. Hsu, "A knowledge-based evolutionary algorithm for the multiobjective vehicle routing problem with time windows," Computers and Operations Research, vol. 45, pp. 25-37, 2014.

[13] B. Ombuki, B. J. Ross, and F. Hanshar, "Multi-objective genetic algorithms for vehicle routing problem with time windows," Applied Intelligence, vol. 24, no. 1, pp. 17-30, 2006.

[14] D. Pisinger and S. Ropke, "A general heuristic for vehicle routing problems," Computers \& Operations Research, vol. 34, no. 8, pp. 2403-2435, 2007.

[15] H. I. Calvete, C. Galé, M.-J. Oliveros, and B. Sánchez-Valverde, "A goal programming approach to vehicle routing problems with soft time windows," European Journal of Operational Research, vol. 177, no. 3, pp. 1720-1733, 2007.

[16] S. Elloumi and P. Fortemps, "A hybrid rank-based evolutionary algorithm applied to multi-mode resource-constrained project scheduling problem," European Journal of Operational Research, vol. 205, no. 1, pp. 31-41, 2010. 


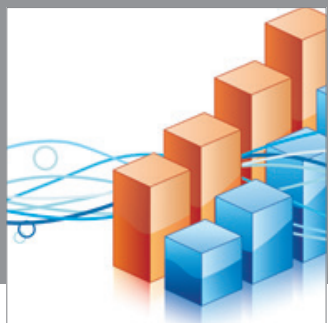

Advances in

Operations Research

mansans

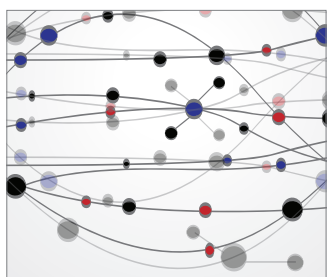

The Scientific World Journal
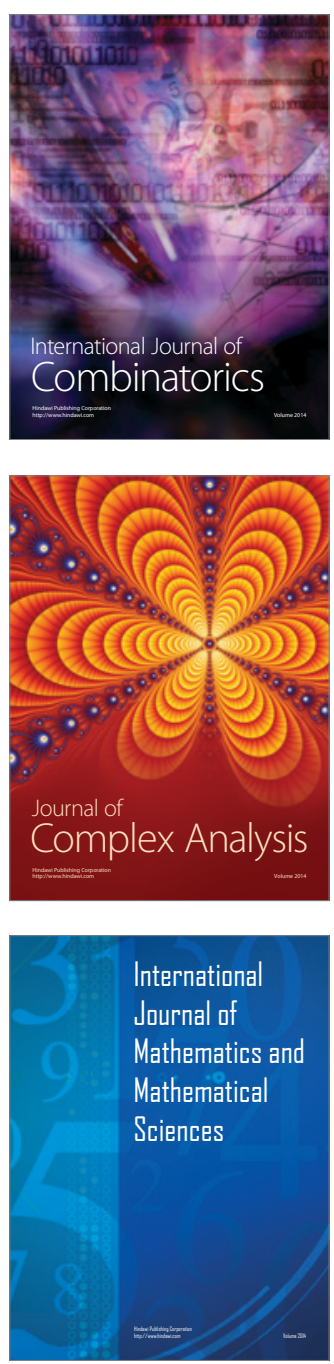
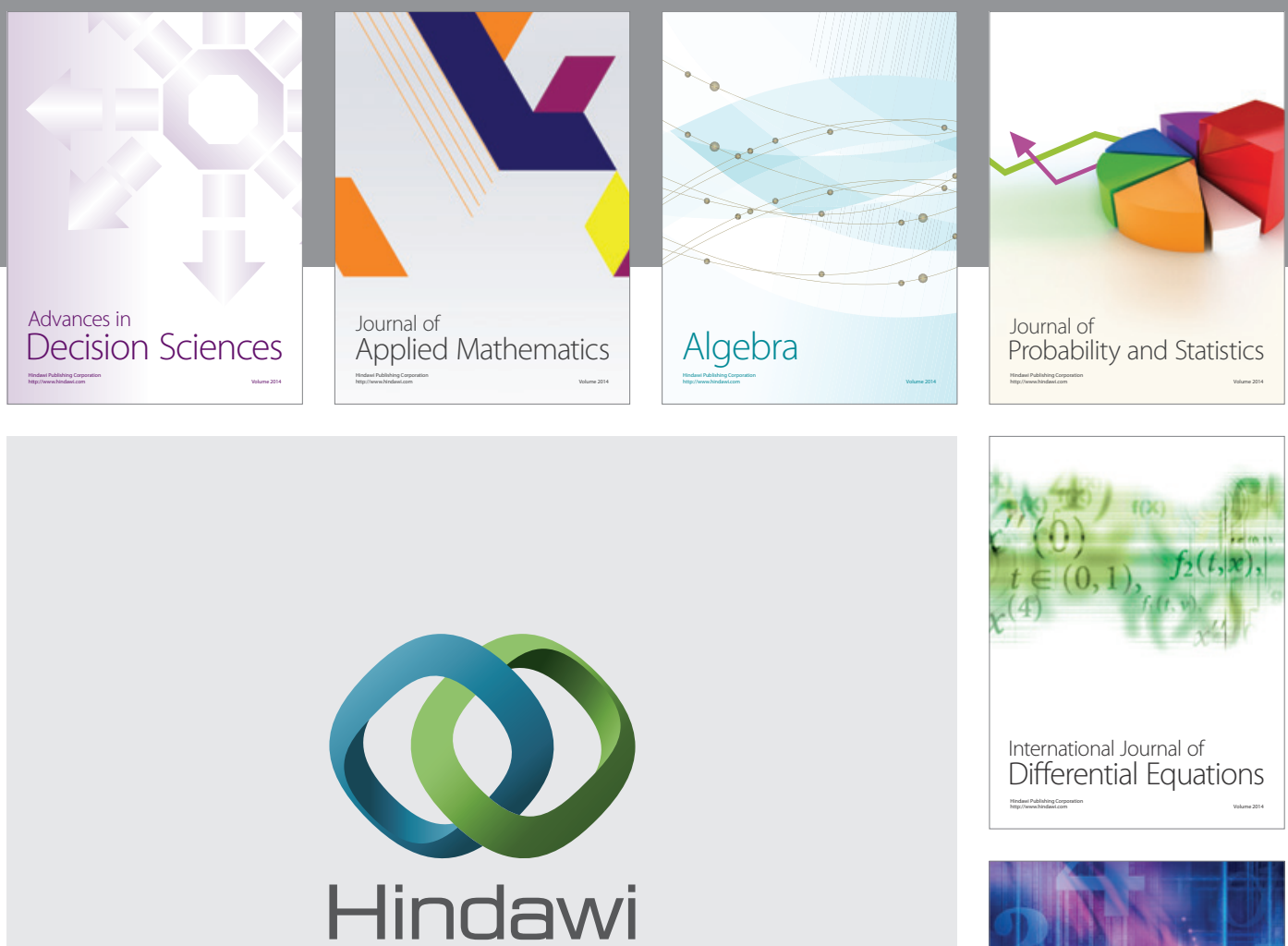

Submit your manuscripts at http://www.hindawi.com
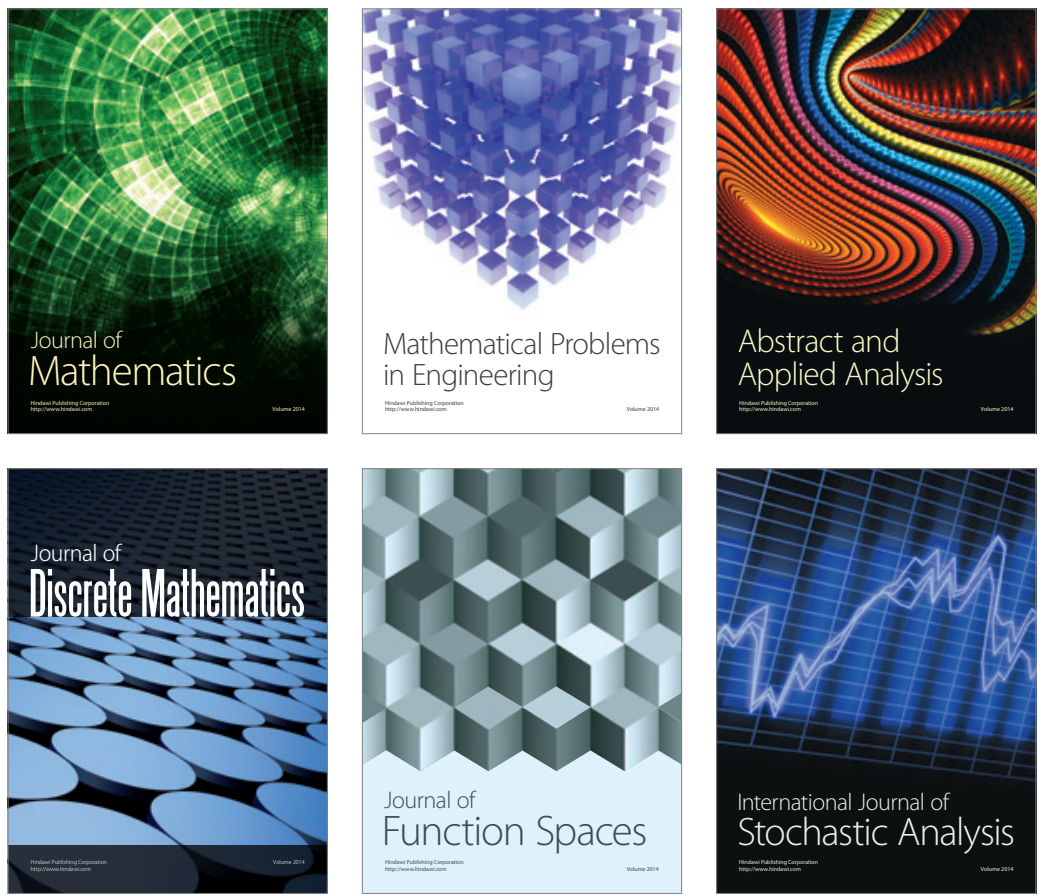

Journal of

Function Spaces

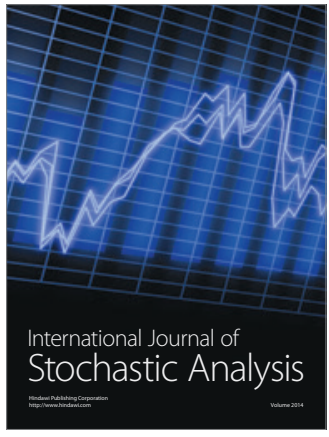

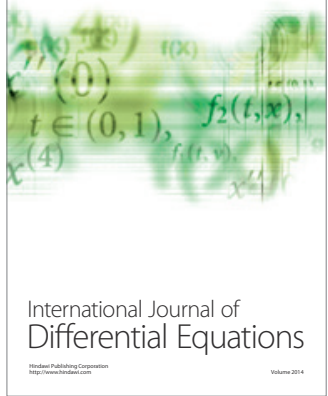
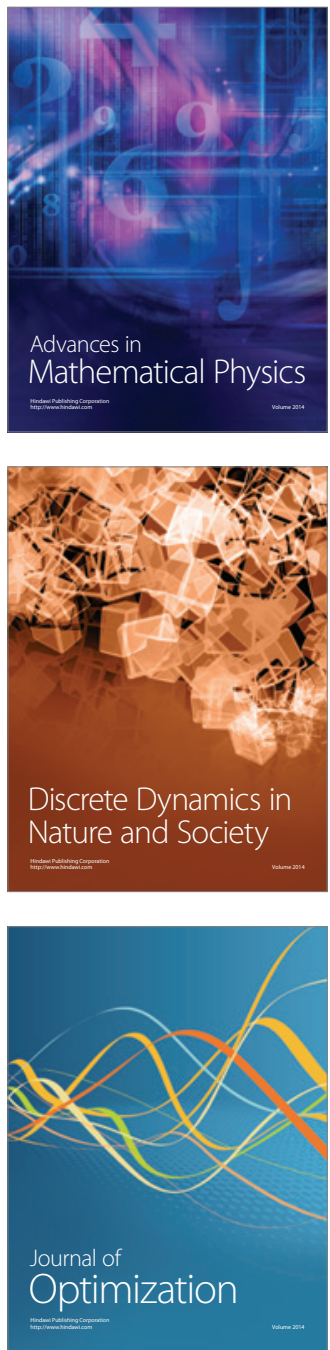